\title{
EFFECT OF REYNOLDS NUMBER ON THE EDDY STRUCTURE IN A LID-DRIVEN CAVITY
}

\author{
T.P. CHIANG, W.H. SHEU* AND ROBERT R. HWANG \\ Department of Naval Architecture and Ocean Engineering, National Taiwan University, 73 Chou-Shan Road, \\ Taipei, Taiwan
}

\section{SUMMARY}

In this paper we apply a finite volume method, together with a cost-effective segregated solution algorithm, to solve for the primitive velocities and pressure in a set of incompressible Navier-Stokes equations. The well-categorized workshop problem of lid-driven cavity flow is chosen for this exercise, and results focus on the Reynolds number. Solutions are given for a depth-to-width aspect ratio of 1:1 and a span-to width aspect ratio of 3:1. Upon increasing the Reynolds number, the flows in the cavity of interest were found to comprise a transition from a strongly two-dimensional character to a truly three-dimensional flow and, subsequently, a bifurcation from a stationary flow pattern to a periodically oscillatory state. Finally, viscous (Tollmien-Schlichting) travelling wave instability further induced longitudinal vortices, which are essentially identical to Taylor-Görtler vortices. The objective of this study was to extend our understanding of the time evolution of a recirculatory flow pattern against the Reynolds number. The main goal was to distinguish the critical Reynolds number at which the presence of a spanwise velocity makes the flow pattern become three-dimensional. Secondly, we intended to learn how and at what Reynolds number the onset of instability is generated. (c) 1998 John Wiley \& Sons, Ltd.

KEY WORDS: lid-driven cavity; Taylor-Görtler-like vortices; instabilites

\section{INTRODUCTION}

With the advent of high-speed computers, numerical simulation of flow physics has received increasing acceptance as a practical method in research institutes and industry. A desirable attribute of the computational fluid dynamics (CFD) technique is its flexibility when conducting parametric studies. It is in this light that we have been stimulated to explore in depth numerically the effect of the Reynolds number on the flow physics in a lid-driven rectangular cavity.

Research into the lid-driven cavity flow structure is an area of continuing interest and was selected for a benchmark study in a major international workshop [1]. This classical problem has attracted considerable attention because its flow configuration is relevant to many industrial applications. It is its geometrical simplicity which facilitates experimental calibrations or numerical implementations, thus providing benchmark data for comparison and validation. Inside this cavity, however, the flow physics is by no means simple. Several flow characteristics which prevail in processing industries, such as boundary layers, eddies of

\footnotetext{
* Correspondence to: Department of Naval Architecture and Ocean Engineering, National Taiwan University, 73 Chou-Shan Road, Taipei, Taiwan.

CCC 0271-2091/98/050557-23\$17.50

Received December 1995

(C) 1998 John Wiley \& Sons, Ltd.

Revised May 1996
} 
different sizes and characteristics and various instabilities, may coexist. Advancing our understanding of the flow evolution in a cavity is thus of importance in acquiring more physical insight into industrial flows. In the past three decades there have been substantial developments which have extended our understanding of the evolution to unsteadiness, instability and turbulence.

Numerical investigation of the flow physics inside a lid-driven cavity dates back to the pioneering work of Burggraf [2], followed by quite a few two-dimensional analyses. While we become aware of large-scale flow characteristics prevailing in the cavity through these two-dimensional analyses, physical subtleties are still inaccessible, because realistic flows are three-dimensional in nature. More recently, fueled by technological advances in computer hardware, the use of three-dimensional numerical simulation as a tool for investigating physical phenomena has been growing steadily. In a parallel development, experimental calibrations on shear-driven cavity flow appeared in the early 1980s. A literature review on the lid-driven cavity flow problem, experimental or numerical, remains insufficient, to the exclusion of the research work of Street and his colleagues [3-12]. In fact, experimental visualization and numerical prediction of Taylor-Görtler-like (TGL) vortices were first accomplished at Stanford University by Koseff et al. [4] and Freitas et al. [8] respectively. The major content of this survey will be focused mainly on the work of Koseff and Street [5-7], Freitas et al. [8] and Freitas and Street [9]. Experimental investigation of cavity flow for Reynolds numbers between $10^{3}$ and $10^{4}$ started in the early 1980s. From 1982 to 1984, Koseff and Street [3-7] addressed values of SAR $(\equiv L: B)=1,2$ and 3 . They observed not only corner vortices in the vicinity of the two vertical end-walls but also locally spreading TGL vortices. In the case $R e \approx 3000$, eight pairs of TGL vortices were observed. At $R e \approx 6000$, three more pairs (i.e. a total of 11 pairs) of TGL vortices become visible. For Reynolds numbers as high as 6000-8000, regular unsteadiness is no longer sustained and thus evolves into turbulence. Spiralling spanwise motion has been discussed mainly inside the downstream secondary eddy (DSE). To our knowledge, none of the previous studies has dealt with the flow physics in the upstream secondary eddy (USE). In 1988-1989, Reynolds numbers in the range 3200-10 000 were considered by Prasad and Koseff [10], who took values of SAR $=1: 2,2: 3,5: 6$ and 1:1 into consideration. The emphasis of their study was on investigating the influence of the reduced SAR on the increase in end-wall viscous drag. More recently, Reynolds numbers classified as low to medium (100-2000) were considered by Aidun et al. [13]. That paper deserves mention because the authors made a distinction between the flow characteristics against the Reynolds number. Prior to $R e=825$ the flow inside the cavity remains steady and symmetric with respect to the half-span of the cavity. For Reynolds numbers between 825 and 925 , disturbances emanating from the symmetry plane propagate periodically towards the two end-walls. The surface separating the DSE from the primary core becomes irregular as the Reynolds number continues to increase to 1000 . A further increase in $R e$ is responsible for the presence of spikes inside the DSE. Beyond $R e=1300$ these irregular spikes finally develop into TGL vortices. Secondary eddies at the upstream side-wall, which we believe are attributable to the formation of TGL vortices, were not discussed. Even though the flow motion at the downstream side-wall is more apparent, we will consider in this paper that the upstream side-wall serves as the unstable surface.

Numerical predictions of a lid-driven cavity, based on the work of the research group led by Street, have been conducted firstly at $R e=3200$ by Freitas et al. [8] and Freitas and Street [9] for $\mathrm{SAR}=3: 1$ and by Perng and Street [11] for $\mathrm{SAR}=1: 1$ and later at higher Reynolds numbers of 7500 and 10000 by Zang et al. [12] for SAR = 1:2. In 1991 the GAMM Committee sponsored a workshop dedicated to numerical simulation of lid-driven cavity flow at $R e=3200$ 
for $\mathrm{SAR}=3: 1$ [1]. To provide some insight into the computed results, Deville et al. [1] summarized and discussed the main features of the solutions obtained by the contributors to this workshop. Comparison studies were conducted on the number of TGL vortex pairs appearing in the transverse direction, on the performance of the computer codes employed and on the CPU time per unit physical time. Surprising to find is that conclusions are quite different among the contributors, not only on the accessibility of flow symmetry but also on the number of pairs of TGL vortices. In recognition of this, we feel that much work needs to be performed in the years ahead. As a result, we consider a Reynolds number much lower than 3200 .

The paper is organized as follows. In Section 2 we begin by introducing the equations of fluid motion for the incompressible case. Subsequently, the underlying finite volume discretization method, together with the segregated solution algorithm and the multidimensional advection scheme, is briefly described. In Section 3, as an analysis tool, we validate the applicability of the employed computer code in simulating Navier-Stokes flows by carrying out an analytic comparison test. In Section 4 we discuss mainly the flow structure and quantify the spanwise variation under increasing Reynolds number. Attention is given to examining in what Reynolds number range the flow under investigation becomes susceptible to the two-dimensional assumption. Also, a question we address here is how and at what Reynolds number the steady flow pattern starts to cause three-dimensional instability.

\section{MATHEMATICAL AND NUMERICAL METHOD}

In the absence of body forces the working equations permitting the analysis of incompressible and viscous fluid flows take the following form for a given Reynolds number, which is defined as $R e=\rho U_{\mathrm{c}} B / \mu$ :

$$
\begin{aligned}
& \frac{\partial u_{i}}{\partial x_{i}}=0 \quad \text { in } \Omega, \\
& \frac{\partial u_{i}}{\partial t}+\frac{\partial}{\partial x_{m}}\left(u_{m} u_{i}\right)=-\frac{\partial p}{\partial x_{i}}+\frac{1}{R e} \frac{\partial^{2} u_{i}}{\partial x_{m} \partial x_{m}} \quad \text { in } \Omega,
\end{aligned}
$$

where $u_{i}(x, y, z, t)$ is the velocity field, $p$ is the static pressure, $\rho$ is the fluid density and $\mu$ is the dynamic viscosity. According to Figure 1, all the lengths in this paper are non-dimensionalized with $B$. Time, velocity and pressure are normalized by $B / U_{\mathrm{c}}, U_{\mathrm{c}}$ and $\rho U_{\mathrm{c}}^{2}$ respectively. Although in the literature there exist several sets of working variables to choose from in a domain $\Omega$ given by $0 \leq x \leq 1,0 \leq y \leq 3,0 \leq z \leq 1$, we prefer to employ the most popular primitive variable formulation for this class of flows, mainly because this setting possesses the closure boundary and initial conditions [14].

In as much as the above velocity-pressure formulation is considered, we are faced with choosing a strategy of either grid staggering [15] or collocation [16] to store working variables. While node-to-node pressure oscillations can be alleviated in both grids, we favour the first strategy regardless of programming complications. The rationale behind this choice is based on the fact that we lack a set of indispensable closure boundary conditions for analyses involving a Poisson-type pressure correction equation. In a staggered grid setting, each primitive variable takes over a node to itself, whereas the pressure node is surrounded by its adjacent velocity nodes. This permits the natural use of finite volume integration for each conservation equation under these circumstances. 
When dealing with the primitive variable form of the incompressible Navier-Stokes equations, we encounter two well-known technical difficulties. The first of these is the removal of the instability problem when a problem of advection dominance is considered. To avoid spurious velocity oscillations and a false diffusion error, which may greatly pollute the flow physics over the entire domain, we advocate the use of the third-order QUICK [17] upwind scheme to discretize non-linear advective fluxes in a non-uniformly discretized domain. Also, prediction errors stemming from the use of one-to-one curvilinear co-ordinate transformation in structured-type discretization are considerable and are hardly avoidable for problems involving complex geometry and highly distorted meshes. We carry out the analysis in a grid system containing rectangular grids to get rid of the potential loss of accuracy resulting from the use of co-ordinate transformation. The transient term is approximated by using the fully implicit differencing scheme.

The second technical difficulty is ensuring satisfaction of discrete divergence-free velocities in an incompressible flow analysis. This constraint condition can be enforced either by using a mixed formulation or by incorporating the incompressibility constraint through multiple-stage equations. In the first class of methods we encounter a much larger linear system. The choice of the pressure correction segregated algorithm in this study comes naturally, because the algorithm can cut down on the storage demands incurred by the mixed formulation. In the present paper the solutions to the finite volume discretization equations are obtained sequentially for the primitive variables. The underlying iterative algorithm is that of SIMPLEC. The rationale behind the algorithmic ideas may be found in Reference [15].

\section{VALIDATION STUDY}

Accurate numerical approximation of a physical system can by no means be assured. Application of a computer code to simulate mathematical or engineering problems is often preceded by analytical assessment of the employed finite volume computer code in simplified settings.

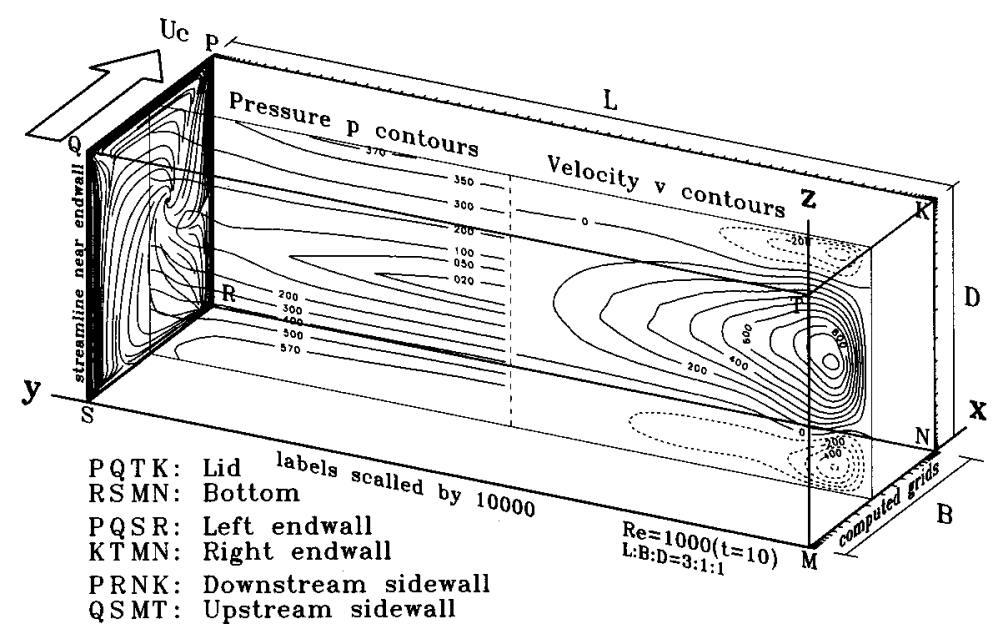

Figure 1. Illustration of rectangular cavity investigated, together with computed pressure gradient, spanwise velocity and end-wall effect 


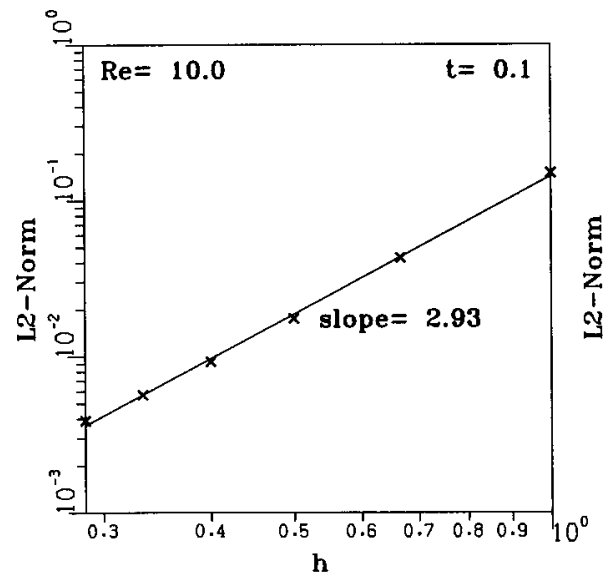

Figure 2. Rate of spatial convergence for three-dimensional scalar equation

In an attempt to verify the flux discretization scheme applied in the present analysis, we consider the analytic problem of Ethier and Steinman [18], subject to the following given velocity $u_{i}(x, y, z, t=0)$, in a simple cubic cavity $(-1 \leq x, y, z \leq 1)$ :

$$
\begin{aligned}
& u=-a\left[\mathrm{e}^{a x} \sin (a y \pm d z)+\mathrm{e}^{a z} \cos (a x \pm d y)\right] \mathrm{e}^{-d^{2} t} \\
& v=-a\left[\mathrm{e}^{a y} \sin (a z \pm d x)+\mathrm{e}^{a x} \cos (a y \pm d z)\right] \mathrm{e}^{-d^{2} t} \\
& w=-a\left[\mathrm{e}^{a z} \sin (a x \pm d y)+\mathrm{e}^{a y} \cos (a z \pm d x)\right] \mathrm{e}^{-d^{2} t}
\end{aligned}
$$

where $a=d / 2=\pi / 4$. To begin with, the QUICK flux discretization scheme has been verified by solving the following scalar equation for $\phi$ :

$$
\frac{\partial\left(u_{i} \phi\right)}{\partial x_{i}}=\frac{1}{R e} \frac{\partial^{2} \phi}{\partial x_{i} \partial x_{i}}+S,
$$

where

$$
S=-\frac{\partial p}{\partial x}-\frac{1}{R e} \frac{\partial \phi}{\partial t}
$$

and

$$
\begin{aligned}
p= & -a^{2} / 2\left[\mathrm{e}^{2 a x}+\mathrm{e}^{2 a y}+\mathrm{e}^{2 a z}+2 \sin (a x \pm d y) \cos (a z \pm d x) \mathrm{e}^{a(y+z)}\right. \\
& \left.+2 \sin (a y \pm d z) \cos (a x \pm d y) \mathrm{e}^{a(z+x)}+2 \sin (a z \pm d x) \cos (a y \pm d z) \mathrm{e}^{a(x+y)}\right] \mathrm{e}^{-2 d^{2} t} .
\end{aligned}
$$

Here the analytic solution for $\phi$ takes the same form as that of $u$ defined in (3).

As usual, we assess here the QUICK-type upwind discretization scheme employed for advective fluxes on the basis of nodal values computed in the uniformly discretized domain and then sum the prediction error in an $L_{2}$-norm sense. With grid spacings being continuously refined in the case of $R e=10$, we can compute the rate of convergence, given $\alpha=\ln \left(\operatorname{err}_{1} / \operatorname{err}_{2}\right) /$ $\ln \left(h_{1} / h_{2}\right)$, from the solutions computed at $t=0.1$ and spatial grid spacings $2 / 2,2 / 3, \ldots, 2 / 6$, $2 / 7$. As indicated by Figure 2, which reveals both the prediction errors and the rate of convergence, namely 2.93, we furthermore perform an analytic study, given by Equations (3) and (5), for working Equations (1) and (2) defined at $R e=1$ in the same cubic cavity. 
According to the finite volume results computed at $t=0-0.1(\Delta t=1 / 160)$, as seen in Figure 3 , the proposed scheme is also applicable to analysis of the incompressible Navier-Stokes equations. Such good agreement with exact solutions provides credibility for the analysis code employed here, and we have sufficient confidence to proceed with the investigation of time-evolving vortices in a rectangular cavity due to the motion of an upper lid.

As mentioned in the Introduction, the flow of a viscous fluid in a three-dimensional cavity driven constantly by a sliding upper plane is a prototype. Here we consider different spanwise ratios, namely (spanwise aspect ratio) SAR $=1$ and 3, Reynolds numbers, namely $R e=400$, 1000 and 3200, for further confirmation of the applicability of the computer code being analytically studied to simulate this problem. The simulation quality was assessed on the basis of available mid-sectional velocity profiles along both vertical and horizontal centrelines. According to the computed finite volume solutions and their convergence histories, as depicted in Figures 4-6, for cavities considered and the Reynolds numbers investigated, the resulting agreement with other numerical solutions of $\mathrm{Ku}$ et al. [19], Kato et al. [20], Cortes and Miller [21], Babu and Korpela [22], Arnal et al. [23] and Kost et al. [24] is close enough.

\section{NUMERICAL RESULTS AND DISCUSSION}

In this section we describe a three-dimensional simulation of the fluid flow in a rectangular cavity defined by $L: B: D=3: 1: 1$ (or SAR = 3:1). The Reynolds number chosen for this cavity is based on the lid speed, the width of the cavity and the kinematic viscosity of the working fluid. At $t=0$ the cavity is subjected to a sudden lid motion at its roof. To avoid ambiguity over whether or not the symmetry of the flow is a feature of the rectangular cavity investigated, as evidenced by the papers presented at the GAMM workshop [1], this problem was simulated in the whole cavity covered with a non-uniform grid of $34 \times 91 \times 34$ resolution. We will address the secondary eddies, the transition from two- to three-dimensionality and that from a steady to an unsteady status in accordance with the increase in Reynolds number. Exploiting the experimentally verified conclusions given in the work of Koseff and Street [5-7], we are led to believe that the laminar flow assumption holds in the range $1<R e<2000$.

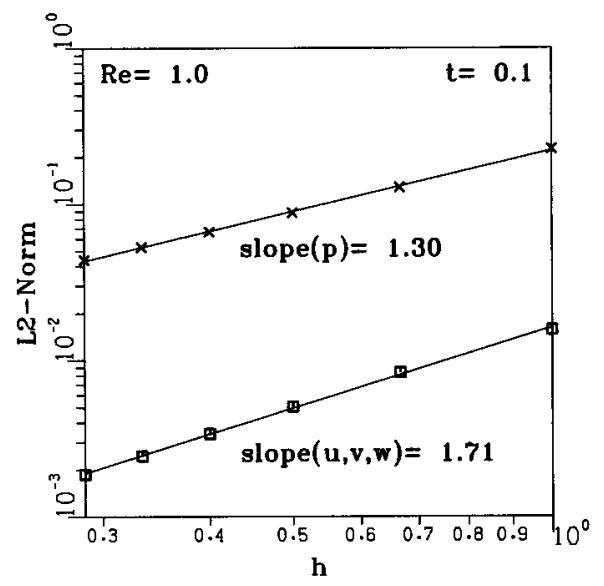

Figure 3. Computed rates of convergence of velocities and pressure for Navier-Stokes equations 


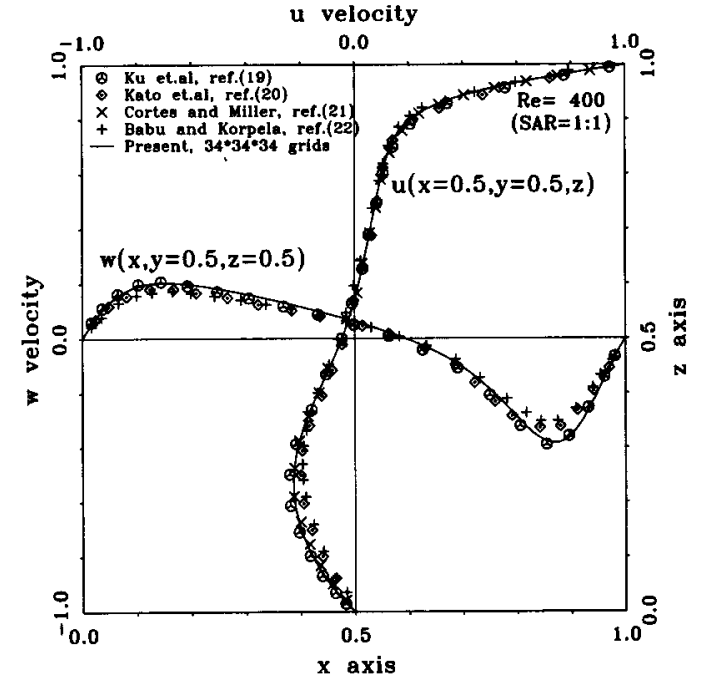

(a)

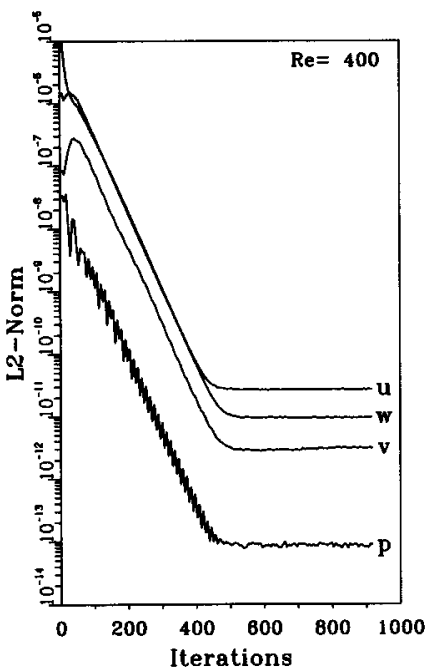

(b)

Figure 4. Comparison study of $\mathrm{SAR}=1: 1$ and $R e=400$ : (a) mid-sectional velocities at symmetry plane $y=0.5$; (b) error reduction plots for working variables

The flow inside the rectangular cavity considered here features a nearly zero spanwise velocity and a flow symmetry at $R e \leq 10$. This is verified in our three-dimensional simulations, as given in Figure 7(a), in that little distinction between the two sets of sectional profiles can be observed. Numerical evidence confirms that the core $(0.2 \leq y \leq 2.8)$ of the cavity is prevailingly symmetric and the spanwise velocities are negligibly small. Upon increasing the Reynolds number, the presence of visible spanwise velocities induced by the pressure gradient,

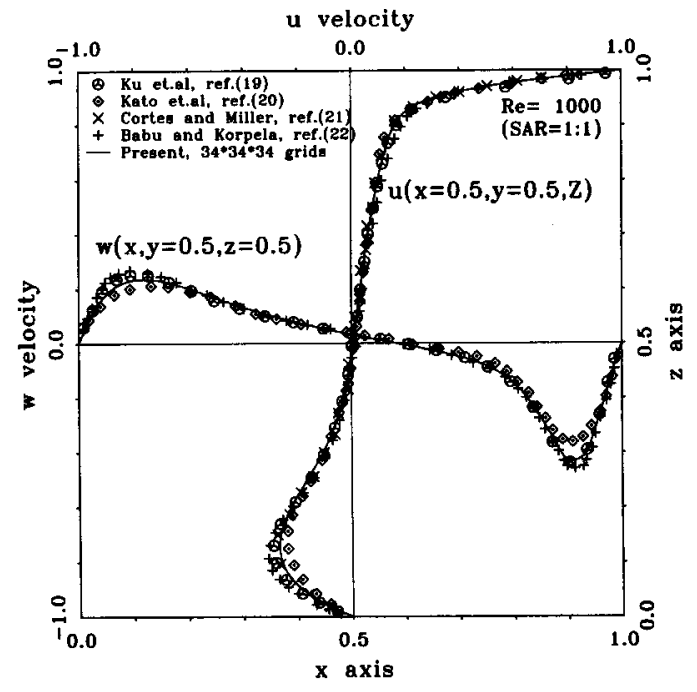

(a)

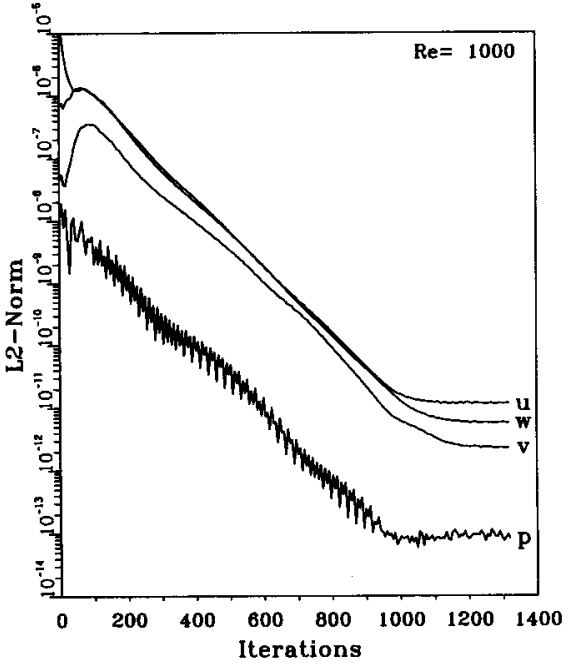

(b)

Figure 5. Comparison study of $\mathrm{SAR}=1: 1$ and $R e=1000$ : (a) mid-sectional velocities at symmetry plane $y=0.5$; (b) error reduction plots for working variables 


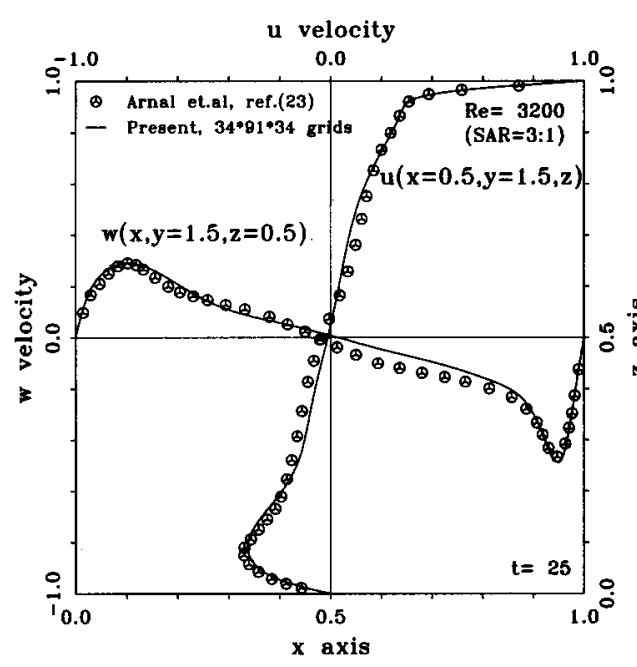

(a)

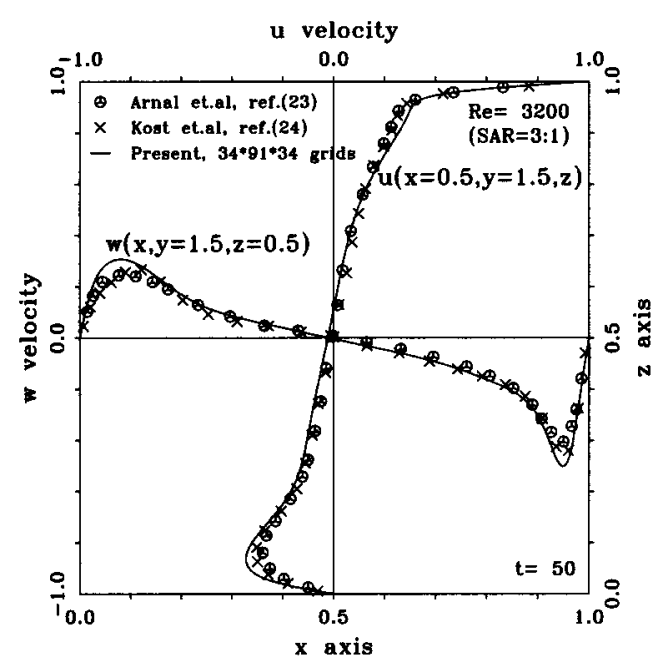

(b)

Figure 6. Comparison study of $\mathrm{SAR}=3: 1$ and $R e=3200$ : (a) mid-sectional velocities at symmetry plane $y=1.5$ $(t=25)$; (b) mid-sectional velocities at symmetry plane $y=1.5(t=50)$

as shown in Figure 1, is a direct result of the two end-walls, which keep the fluid flow from penetrating. The spanwise components show the three-dimensional-like primary character of the fluid flow. Together with the much more dominant two-dimensional flow circulation shown in Figure 8, the rectangular cavity is filled with helixes of different characteristics and sizes. For clear illumination of this spiralling flow structure, it is most suitable to plot its Lagrangian particle tracks. Taking $R e=1000$ for example, we plot in Figure 9 the surface of $v=0$, across which left- and right-running spiralling flows are exclusively apart. Here we focus our attention not only on the particle in the DSE (downstream secondary eddy) (Figure 9(a)) but also on the particle in the USE (upstream secondary eddy) (Figure 9(b)), which has been seldom explored. While both fluid particles migrate towards the end-wall, they differ in the course of their subsequent spiralling motion. The fluid particle designated advances towards the end-wall all the way to the near-wall spanwise location $y=2.95$, as shown in Figure 9(c). According to Figure 9(c), the USE particle is lifted upwards and is then engulfed inwards to the spiral node $\mathrm{N}_{\mathrm{s}}$ via $\overline{\mathrm{cd}}$, followed by a monotonically spiralling motion towards the symmetry plane. As for the DSE particle considered in Figure 9(a), from $\overline{a b}$ it is sucked into the primary core. Comparing the travelling lengths needed for USE and DSE particles to be sucked into the primary core, the shorter length corresponds to the DSE particle. Also, this particle spirals back and forth across the $v=0$ contour surface. In the presence of the increasing spanwise spiralling flow structure, which is an attribute of the increasing Reynolds number, the mid-sectional velocity profiles deviate from those based on two-dimensional analyses $[22,25,26]$. According to Figure 7 , the peak values of the velocity profiles at the symmetry $x-z$ plane are smaller than those obtained on the two-dimensional basis. The decrease in velocity is attributable to the supply of kinetic energy along the spanwise direction. In recognition of the fact that the presence of the spanwise velocity affects the primary flow structure, we have measured the positions of the vortex centres of the core and plotted them against the Reynolds number in Figure 10. Comparing with the vortex centres which are computed based on the two-dimensional solutions of Ghia et al. [26], together with Figures 7(a) and 7(b), we may 

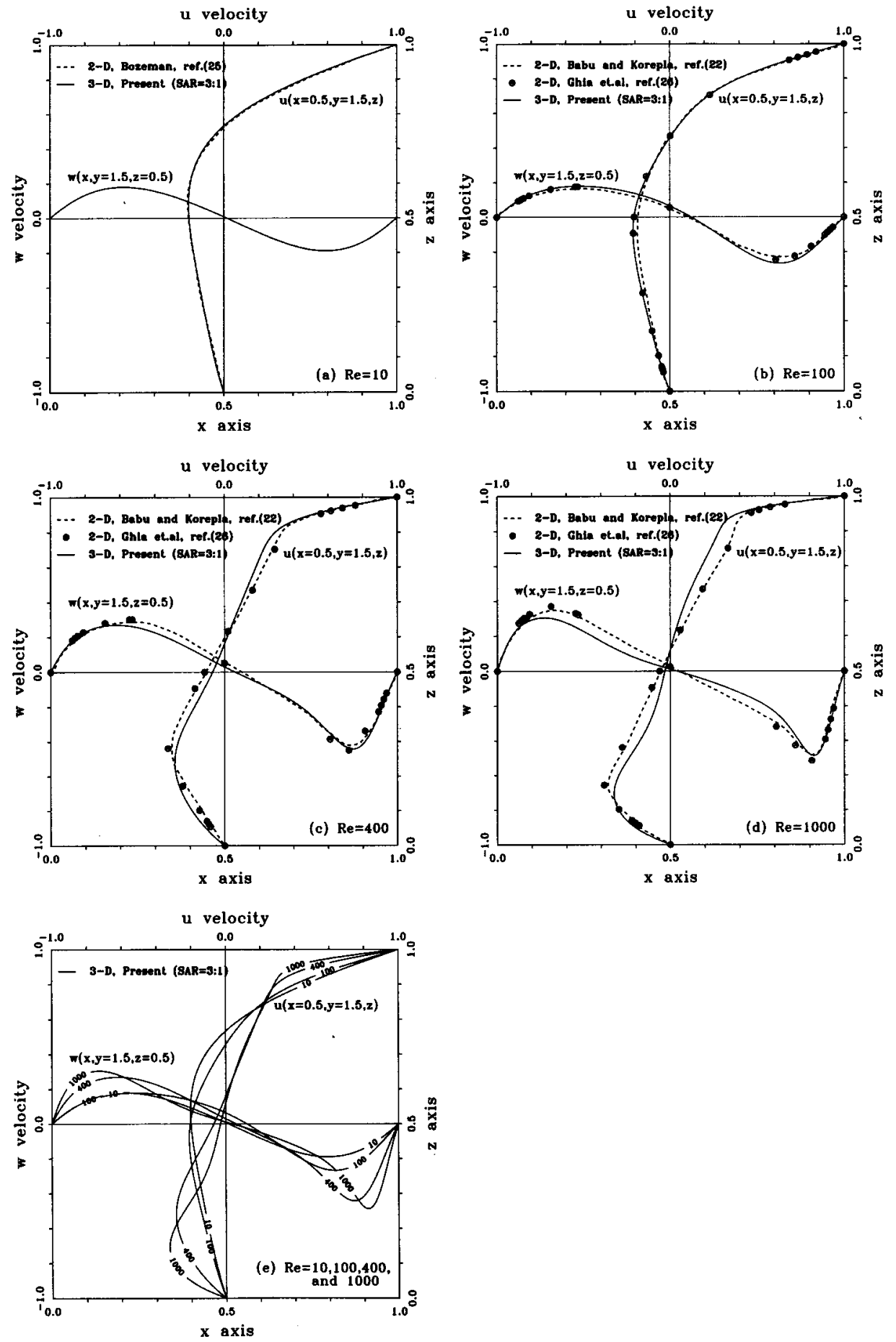

Figure 7. Comparison of present computed velocity profiles at mid-sectional plane with other numerical solutions: (a) $R e=10$; (b) $R e=100$; (c) $R e=400$; (d) $R e=1000$; (e) summary of results given (a) - (d) 
conclude that there is no distinctive variation for a Reynolds number less than 100 . In the three-dimensional analysis conducted at higher Reynolds numbers, the smaller DSE width, as compared with that based on a two-dimensional analysis, is due to the shift of the vortex centre moving towards the bottom wall and the upstream side.

The presence of corner eddies in the cavity is also worthy of study because of their importance in process engineering. Corner eddies are featured by sign changes in the velocity
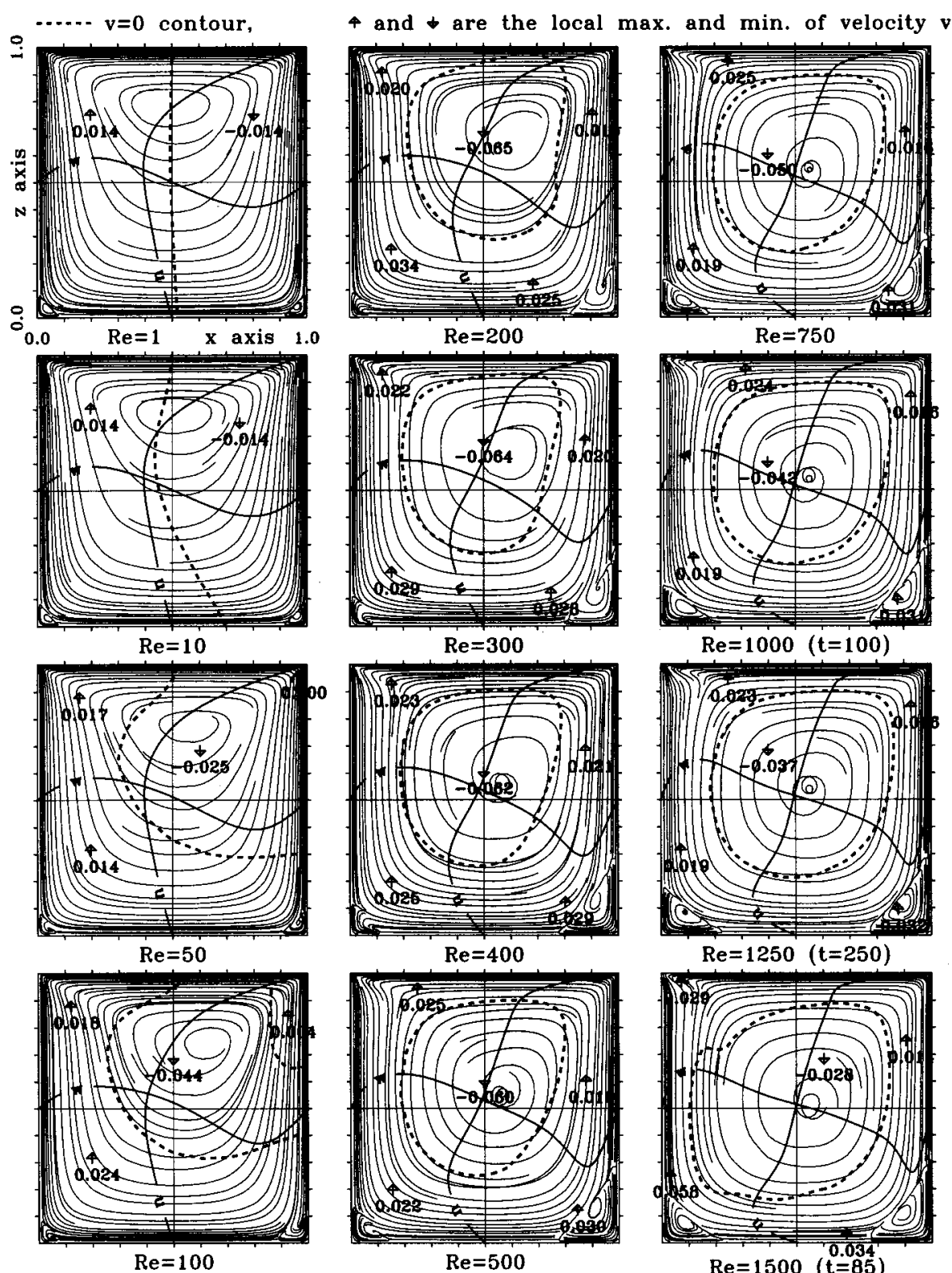

Figure 8. Plots of $u-w$ streamlines, $u-w$ mid-sectional velocity profiles, zero-spanwise-velocity contours and local extreme values of $v$ at plane $y=2.5$ against Reynolds number. For $R e \leq 1000$ the steady state solutions are plotted, whereas for $R e=1250$ and 1500 the plots are at $t=250$ and 85 respectively 

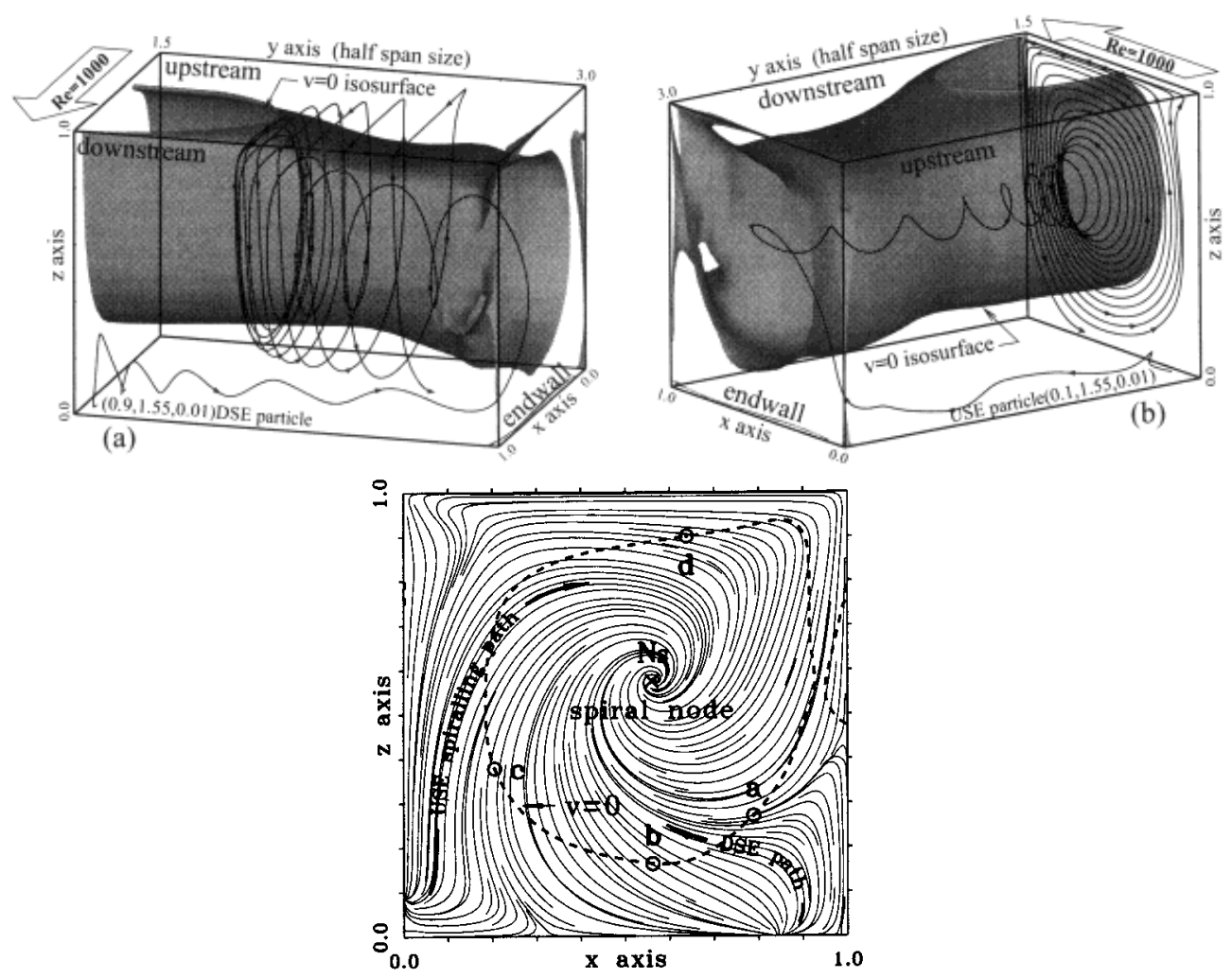

(c)

Figure 9. Illustration of curved zero-spanwise-velocity contours in region $1.5 \leq y \leq 3$ and spiralling particle motions starting from USE at $(0.1,1.55,0.01)$ and from DSE at $(0.9,1.55,0.01)$ : (a) perspective view from downstream side; (b) perspective view from upstream side; (c) planar view of spiralling motion at $y=2.95$

of the flow at the geometric corner. Across the corner eddy the flow is characterized by flow reversals and pressure variations. It is thus worthwhile to examine the influence of the Reynolds number on the upstream and downstream eddies. With this objective in mind, we plot in Figure 11 the eddy sizes at the symmetry plane. Clearly visible from Figure 11 is the trend that the change in eddy size against the Reynolds number follows a curve which is similar to that given by the two-dimensional data of Ghia et al. [26]. Regardless of the presence of three-dimensionality, there is also little variation in values except for the DSE width. The main reason for the much smaller value of the DSE width, as compared with that of its two-dimensional counterparts, can be explained graphically as follows by Figure 12. Along the downstream side-wall the lid-driven downward flow is split into two streams, providing the subsequent USE and DSE spiralling particle motions. As seen in Figure 12(a), spiralling motions of different classes are bisected by the dividing line. For the sake of clarity we denote hereinafter the separation line (or surface) as the border line (or surface) of the primary core and the secondary eddies. Examination of Figure 11 reveals that for Reynolds numbers in the range $200<R e<400$ there exists a change in slope. This is particularly apparent at $R e=300$ in the curve of the DSE height. It is fair to say that as the Reynolds number increases beyond 300 , the flow structure inside the rectangular cavity starts to show new physics. In support of what we mean by new emerging flow physics, we provide the following evidence graphically. 


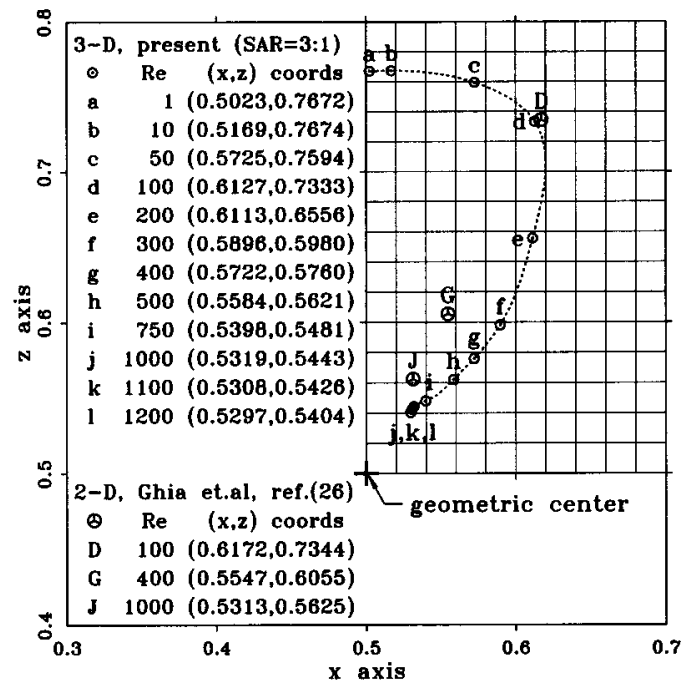

Figure 10. Plot of vortex centres, together with those of Ghia et al. [26], at symmetry plane $y=1.5$ against Reynolds number

On the contour surface of the zero spanwise velocity we begin to observe a free shear roller in Figure 13 as the Reynolds number surpasses 300. According to Figure 8, inside the zero contour of the spanwise velocity the maximum velocity of the spiralling particle motion starts to decrease when $R e>300$ and is accompanied by an enlarged size inner to $v=0$. The decrease in velocity is a direct result of the normal resistance force at the symmetry plane exerted by the two approaching left- and right-running spiral motions. It is also important to point out that, as seen in Figure 12(b), the separation line may detach from the upstream side-wall. The critical Reynolds number leading to such detachment is $R e=300$. On increasing the Reynolds

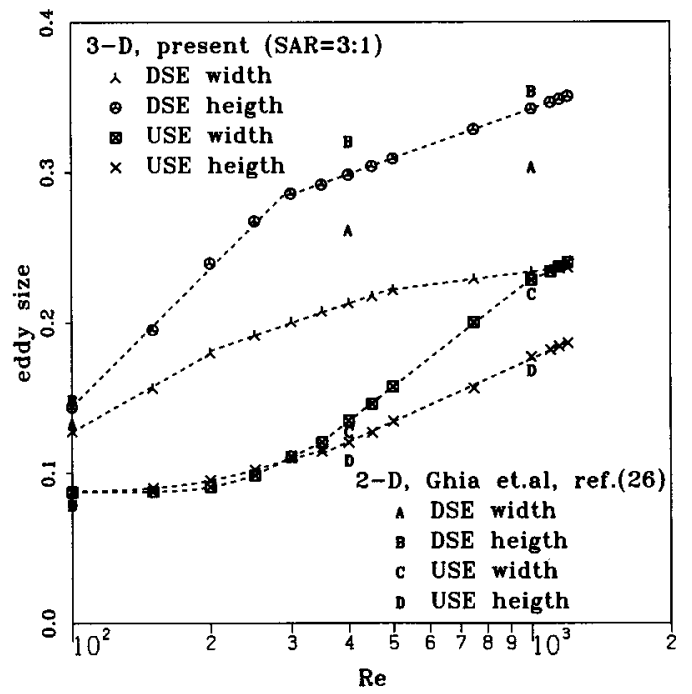

Figure 11. Plot of eddy sizes, together with those of Ghia et al. [26], at symmetry plane $y=1.5$ against Reynolds number. The sizes of the eddies are defined in Figure 12 

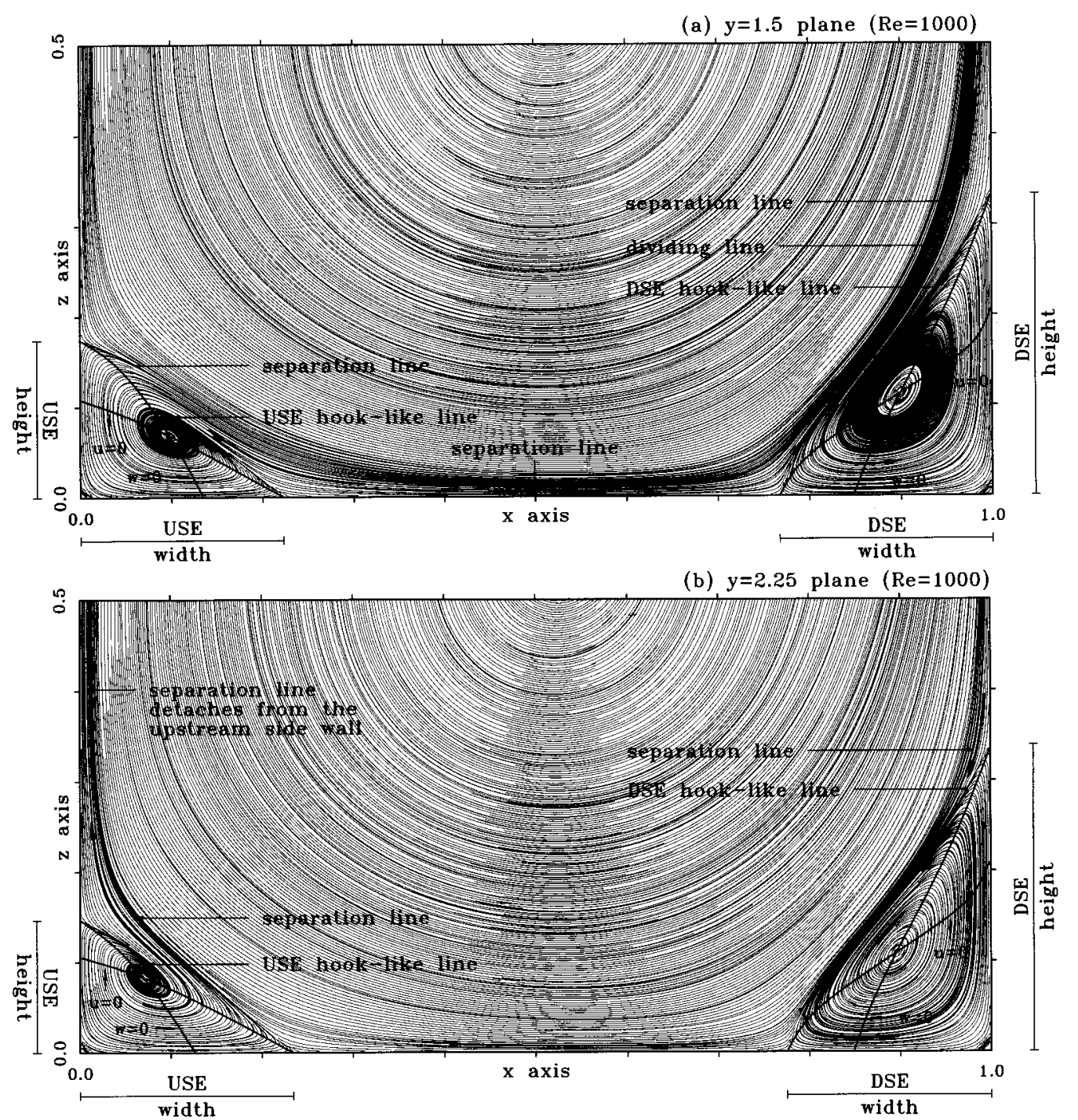

Figure 12. Illustration of interaction of different eddies and definition of eddy sizes for $R e=1000$ at (a) symmetry plane $y=1.5$ and (b) $y=2.25$ plane

number further, the value of the USE width continues to increase at a much faster pace. At $R e \approx 1000$ the value of the USE width approaches that of the DSE width. Afterwards the flow pattern shows unsteadiness, leading to a waving flow structure and finally the presence of Taylor-Görtler-like vortices. This implies that beyond the critical Reynolds number $R e=1000$ the steady state assumption no longer holds. In circumstances where the Reynolds number exceeds this critical value, the shape of the contour line of $v=0$, as shown in Figure 8, approaches that of the nearby streamlines. This alignment retards the transport processes cutting across the $v=0$ surface. As a result, the flow system under investigation becomes destabilized, as viewed from the distorted contour surface of $v=0$ in Figure 8 at $R e=1500$. 
For the sake of completeness we summarize in Figure 14 the eddy sizes against the Reynolds number. For Reynolds numbers in the range $100<R e<1200$ the plots shown in Figure 14 do not vary with time in our study. This implies that the sizes of the USE and DSE can be regarded as being steady for Reynolds numbers below 1200. Close examination of the eddy sizes shown in Figure 14 reveals that the rate of increase in the size of the DSE decreases when
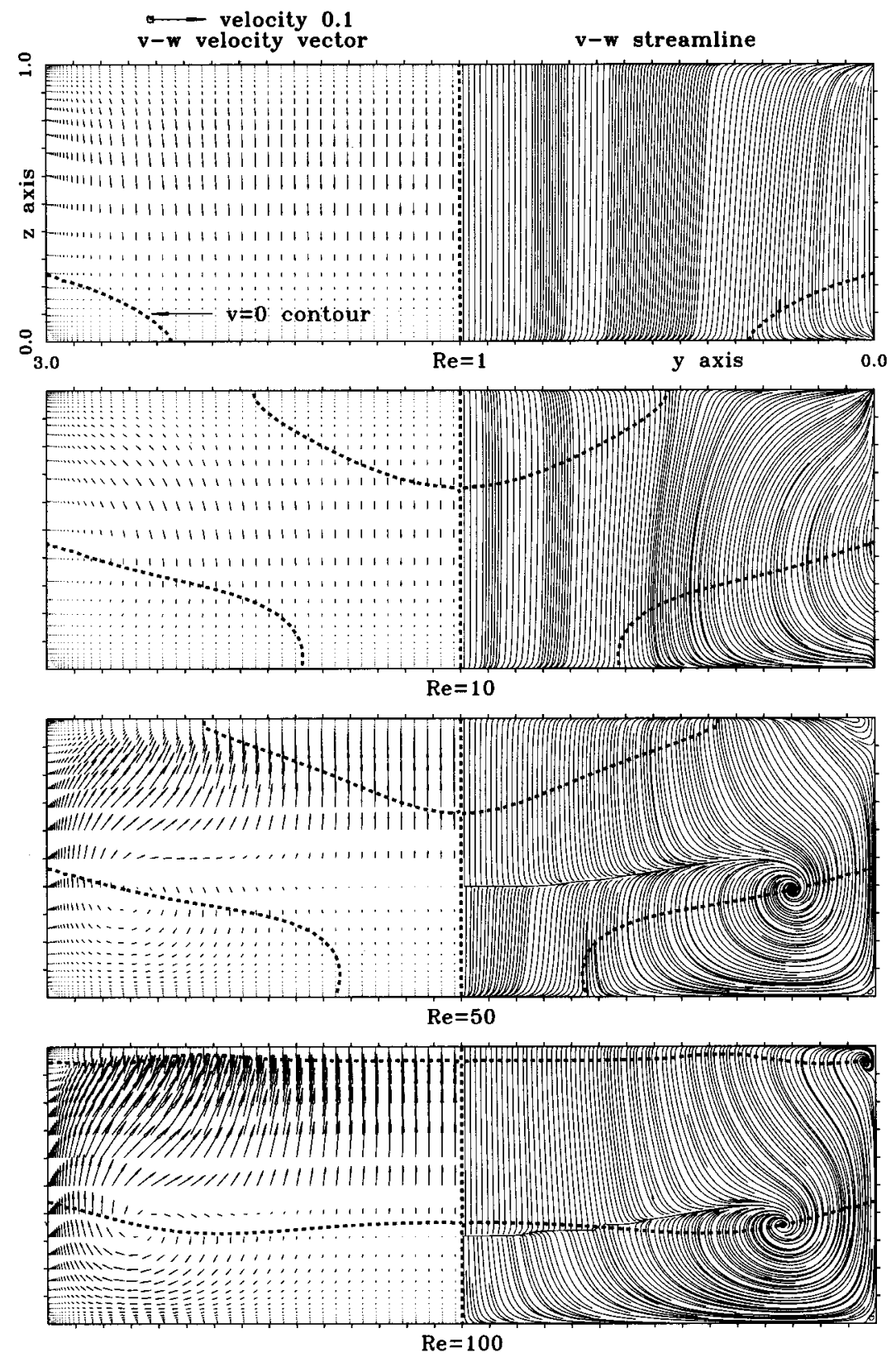

Figure 13. Variation of $x$-plane $(x=0.525)$ flow structures and zero-spanwise-velocity contours against Reynolds number 

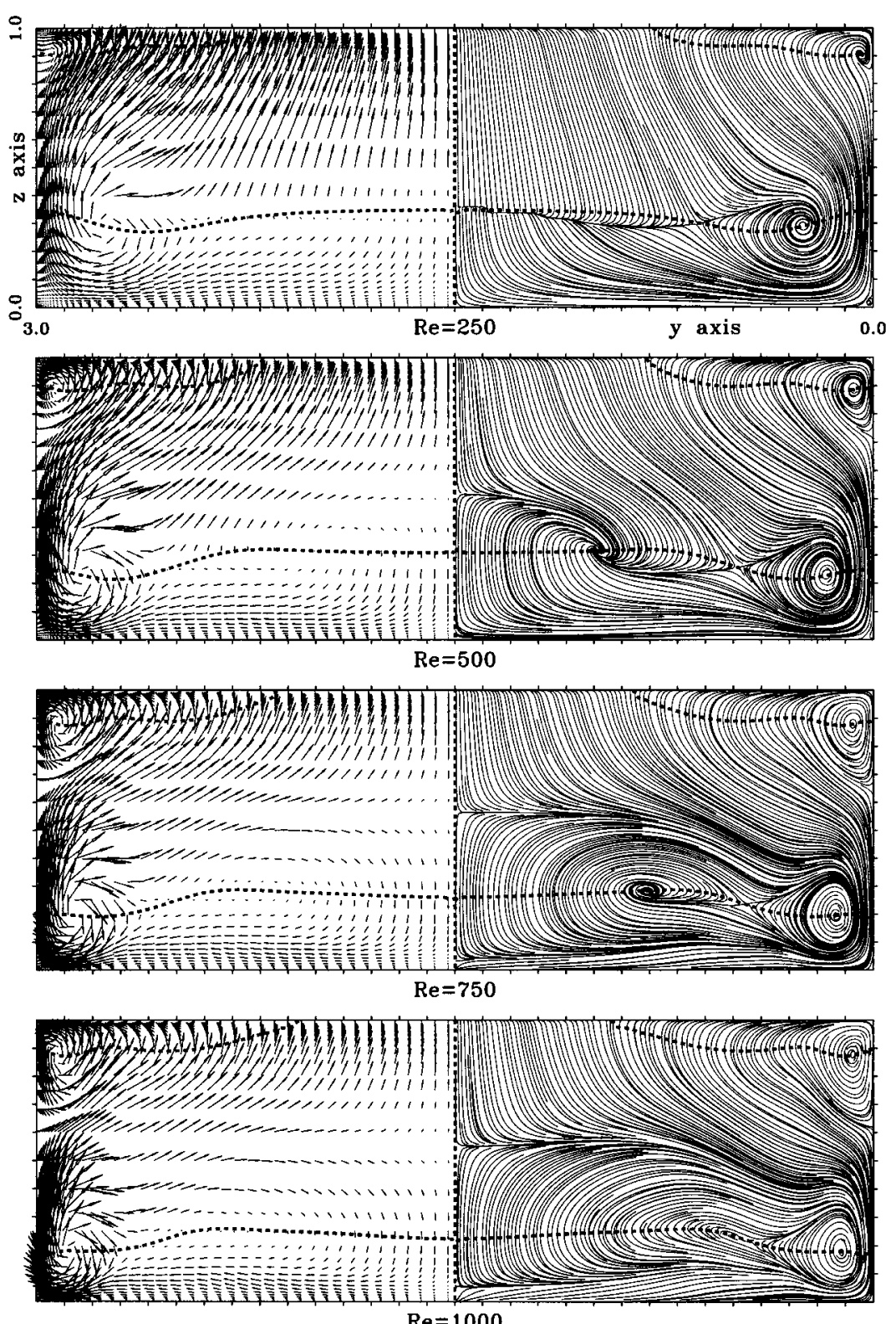

Figure 13 (Continued)

$R e>300$, as opposed to the trend for the size of the USE. As expected, the eddy sizes plotted in Figure 14 approach asymptotically different values in the direction towards the symmetry plane, no matter which Reynolds number is investigated. These constants correspond to the eddy sizes computed based on two-dimensional calculations. Also clearly seen in Figure 14 is a sharp decrease in the DSE width and USE height, together with a sharp increase in the DSE height and USE width, in regions near the end-wall, as viewed along the direction towards the end-wall. Such an appreciable variation is due to the presence of the upstream side-wall at 
which the separation surface detaches, denoted by ' $E$ ' in Figure 14. In the region defined by $1.5<y<2.5$, both the height and width of the DSE remains fairly constant. In contrast with the size of the downstream secondary eddy, there exists clear waviness, with regard to the USE height in particular, as the Reynolds number surpasses 300. This kind of wavy profile is attributable to the detachment (Figure 12(b)) of the separation surface on the upstream side, as depicted by ' $\alpha$ ' in Figure 14 or Figure 15. In response to this detachment, the width increases while the height of the USE decreases, no matter what Reynolds number is under investigation.

In an attempt to clarify at what Reynolds number the lid-driven cavity system starts to show unsteady transport processes, we have plotted the eddy sizes for both secondary eddies against the span in a half-cavity. From careful examination of these plots in Figure 16, which correspond to $t=80-280$, for Reynolds numbers larger than 1200, we regard the flow system at $R e=1250$ as being unsteady. This figure clearly shows that the DSE is much more stable than its USE counterpart, in that the eddy sizes do not vary with time. To illuminate the unsteady aspect of the upstream secondary eddy, close-up plots of the region within the broken line in Figure 16(a) are shown at three time intervals, namely $80 \leq t \leq 120,160 \leq t \leq 200$, and $240 \leq t \leq 280$, in Figures 16(b)-16(d) respectively. From these time-varying plots for both the width and height of the USE, we realize that these wavy profiles tend to move towards the
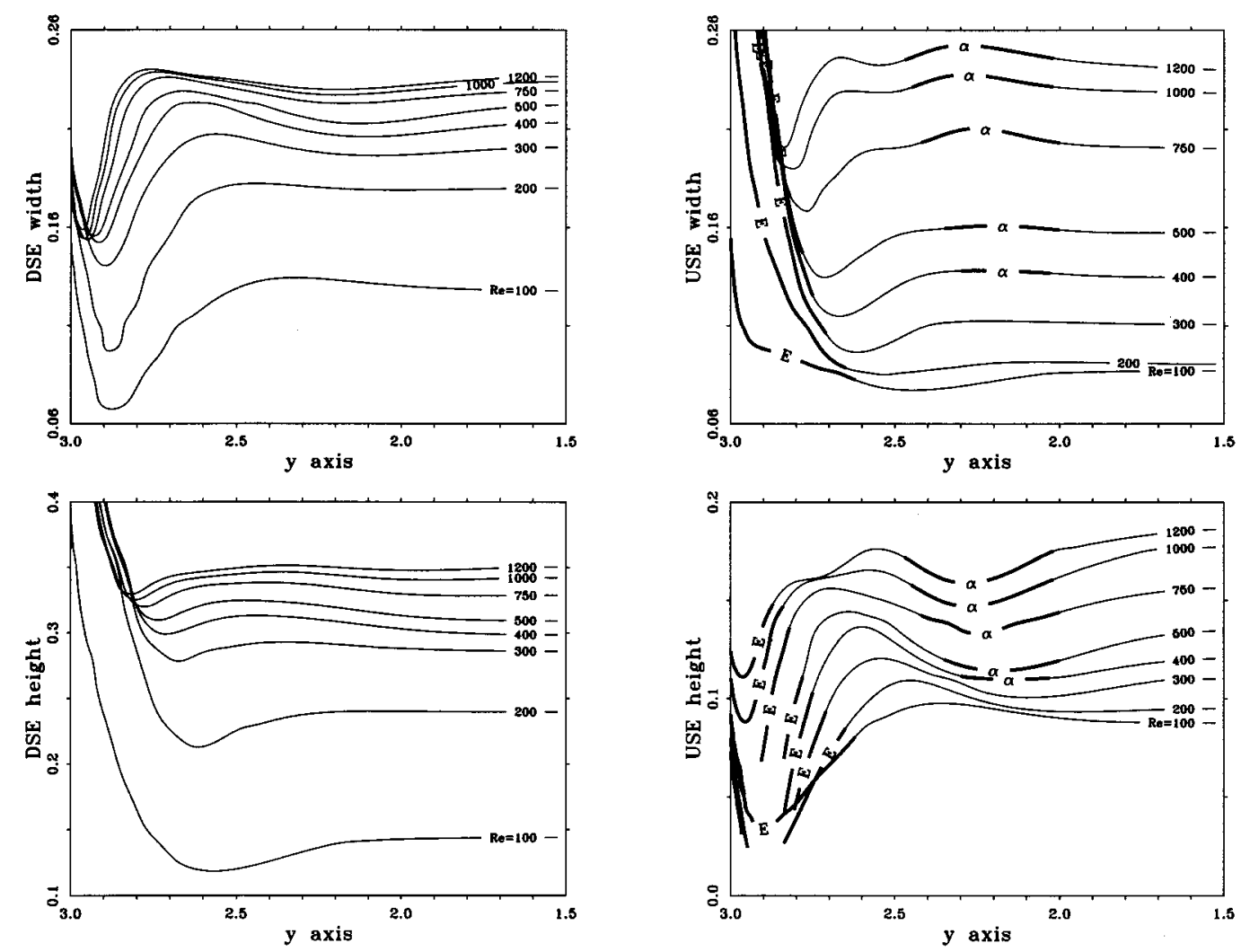

Figure 14. Eddy size variation against span for Reynolds numbers $100 \leq R e \leq 1200$ in half-cavity $1.5 \leq y \leq 3$. 'E' and ' $\alpha$ ' represent regions where the separation surfaces plotted in Figure 15 detach from the upstream surface near the end-wall and other spanwise locations (Figure 12(b)) respectively 

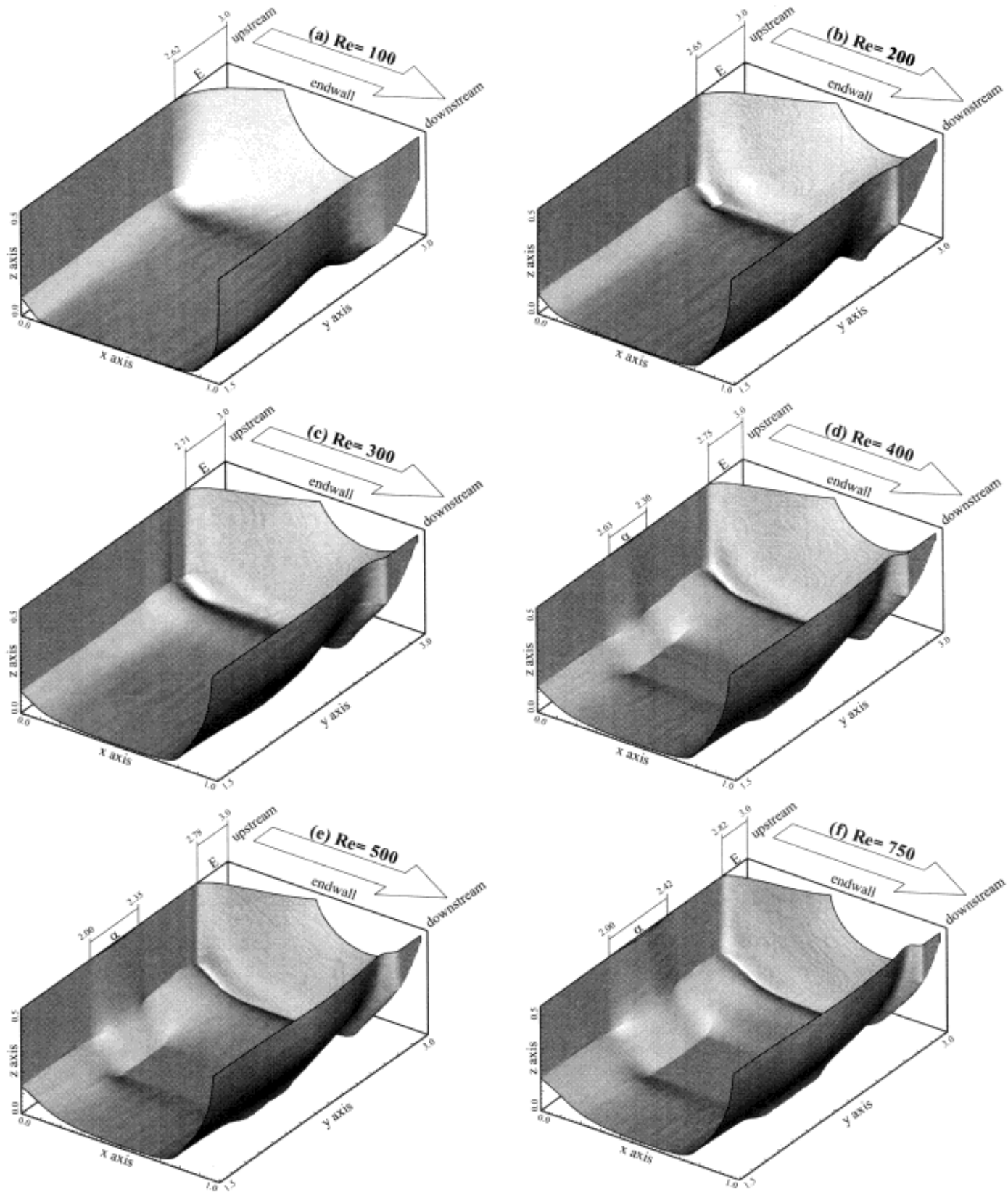

Figure 15. Illustration of surfaces which separate primary core from secondary eddies in quarter-cavity defined by $0 \leq z \leq 0.5,1.5 \leq y \leq 3$; (a) $R e=100$; (b) $R e=200$; (c) $R e=300$; (d) $R e=400$; (e) $R e=500$; (f) $R e=750$; (g) $R e=1000$. The definitions ' $\mathrm{E}$ ' and ' $\alpha$ ' are given in Figure 14

end-wall. The time period is estimated to be 40 and the amplitudes of these heights and widths seem to increase mildly.

In Figure 15, regions denoted by ' $\alpha$ ' become visible at $R e=400$. The presence of such upstream detachment implies the onset of flow unsteadiness. This flow detachment has a close 


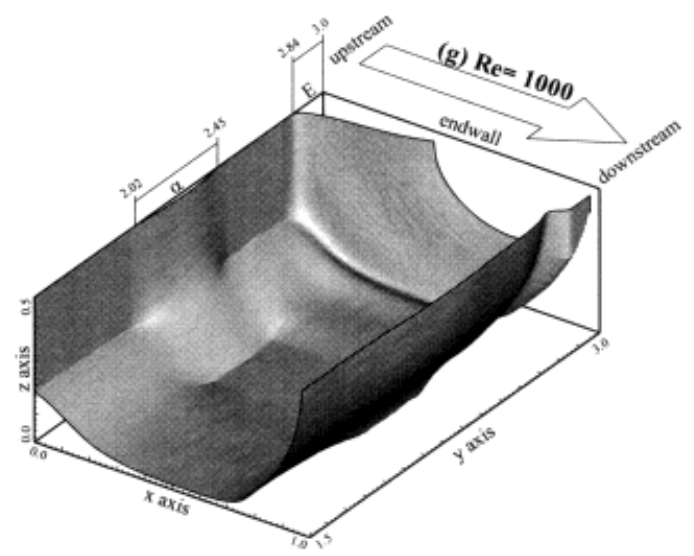

Figure 15 (Continued)

relevance to the formation of TGL vortices. For the sake of completeness we also assign ' $\alpha$ ' in Figure 14. We have tracked the velocity variations at a point, namely $(0.056,2.25,0.315)$, falling within this region for a Reynolds number in the vicinity of 1200 . For Reynolds numbers
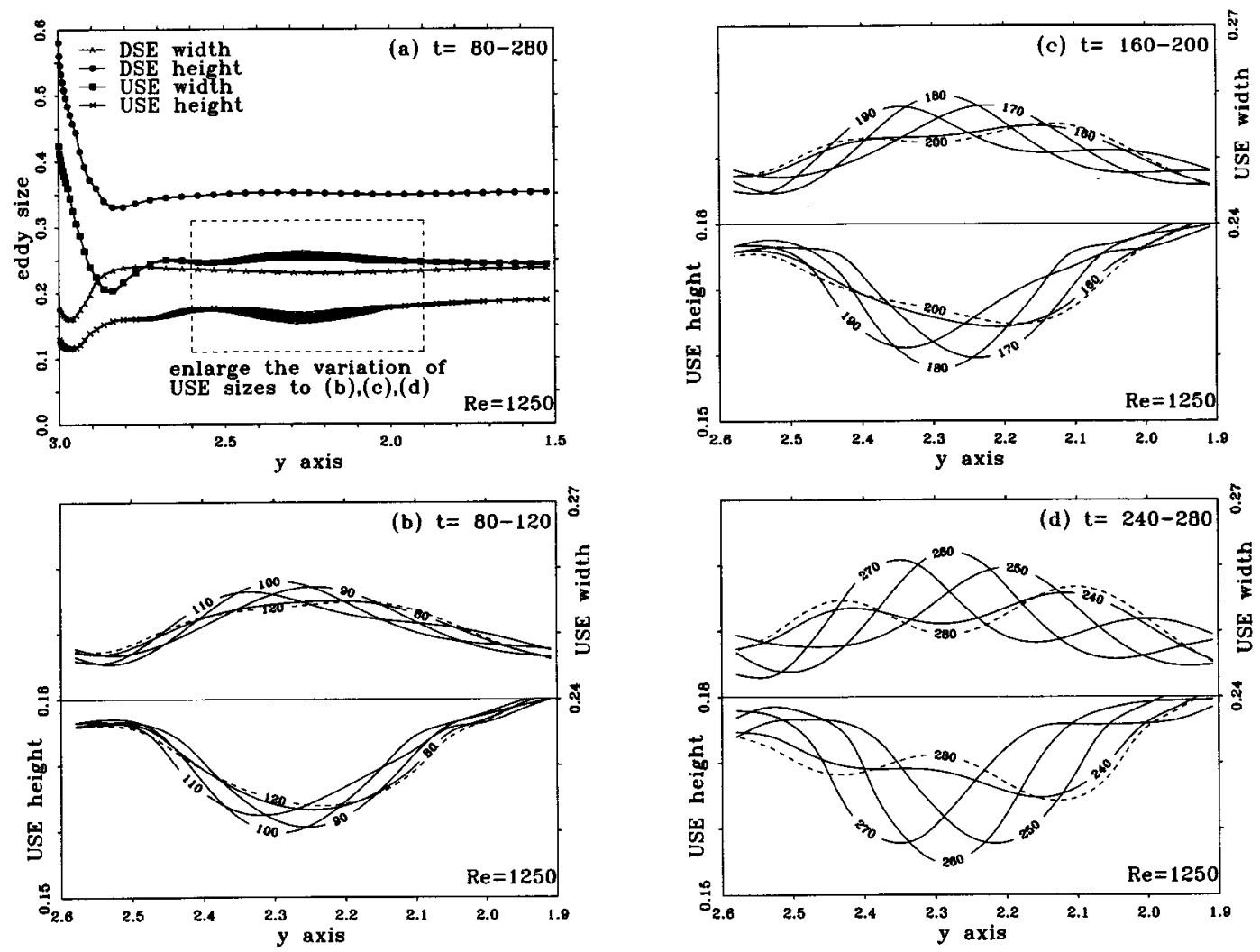

Figure 16. Variation of eddy size against $y$ in half-cavity $1.5 \leq y \leq 3$ for $R e=1250$ : (a) distribution of eddy sizes in time interval $80 \leq t \leq 280$; (b)-(d) close-ups of (a) in region $1.9 \leq y \leq 2.6-$ (b) $80 \leq t \leq 120$; (c) $160 \leq t \leq 200$; (d) $240 \leq t \leq 280$ 


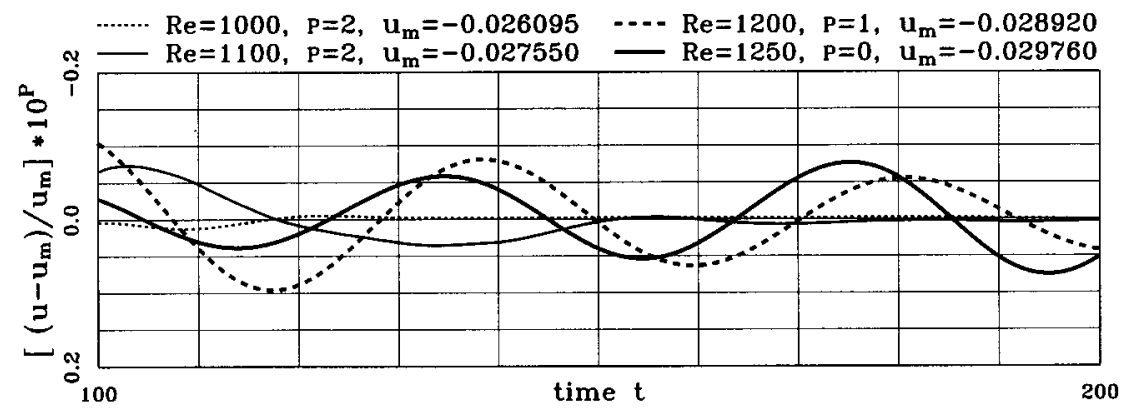

(a) $\left(u-u_{m}\right) / u_{m}$

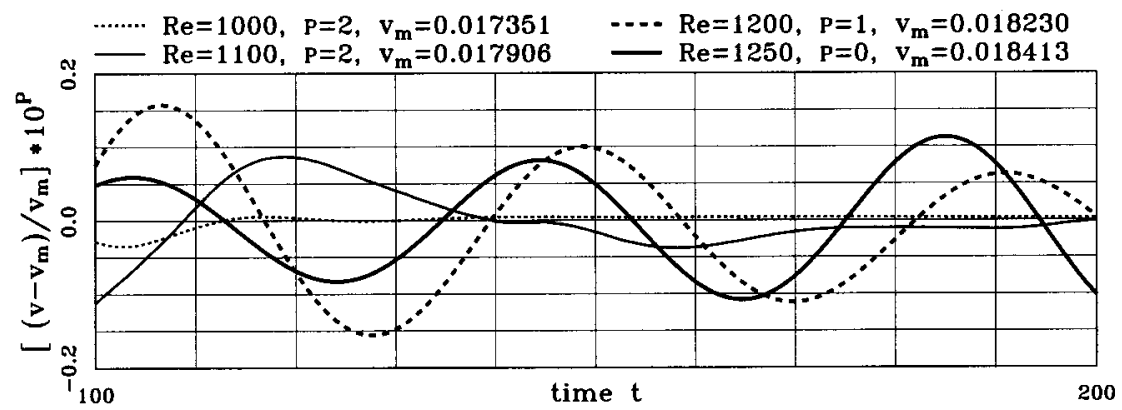

(b) $\left(v-v_{m}\right) / v_{m}$

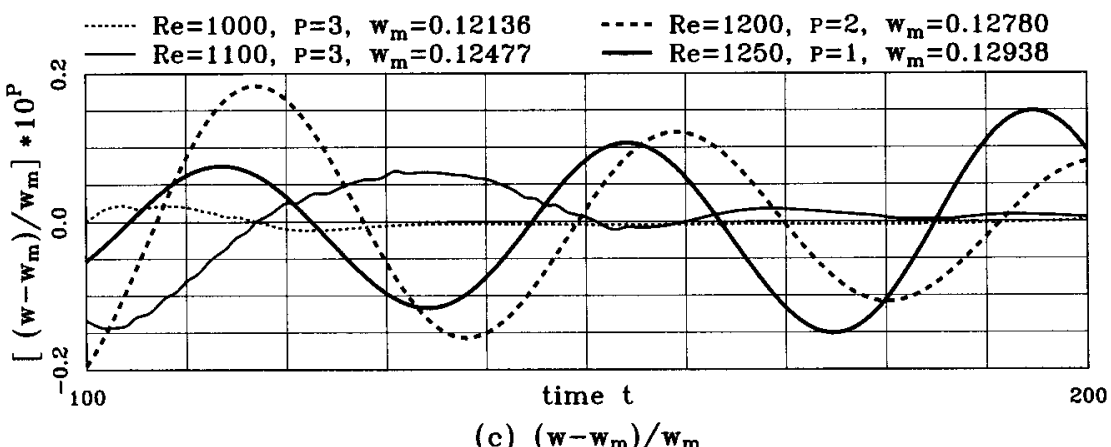

Figure 17. Plots of relative velocity fluctuations at $(0.056,2.25,0.315)$ against time for $100 \leq t \leq 200$ : (a) $\left(u-u_{\mathrm{m}}\right) / u_{\mathrm{m}}$; (b) $\left(v-v_{\mathrm{m}}\right) / v_{\mathrm{m}}$; (c) $\left(w-w_{\mathrm{m}}\right) / w_{\mathrm{m}}$. The subscript ' $\mathrm{m}$ ' denotes the mean quantities based on the values computed at $100 \leq t \leq 200$

of 1000, 1100, 1200 and 1250 we plot in Figure 17 the relative velocity fluctuation against time. As expected, the magnitudes of the fluctuations increase the Reynolds number. For $R e=1000$ and 1100 the fluctuation in the velocity can be regarded as invariant with time. At a higher Reynolds number, $R e=1200$, the relative magnitudes of the velocity components start to fluctuate with a period of 40 . In the wavy profiles the amplitude decays with time for a Reynolds number of 1200, while it grows for a higher Reynolds number of 1250. This increased amplitude paves the way for the onset of flow instability. Also shown in this figure is that the extreme values of $u$ and $w$ coincide roughly with the inflection point of the spanwise velocity component. This velocity setting is akin to the velocity distribution of TGL vortices. 
Increasing continuously the Reynolds number to 1300, TGL vortices burst in the already unsteady flow system, as shown in Figure 18, at time $t>150$. The onset of TGL vortices is due mainly to the increased energy of the disturbances and has been experimentally verified by the work of Aidun et al. [13]. We have examined the values of the velocity component $w$ at

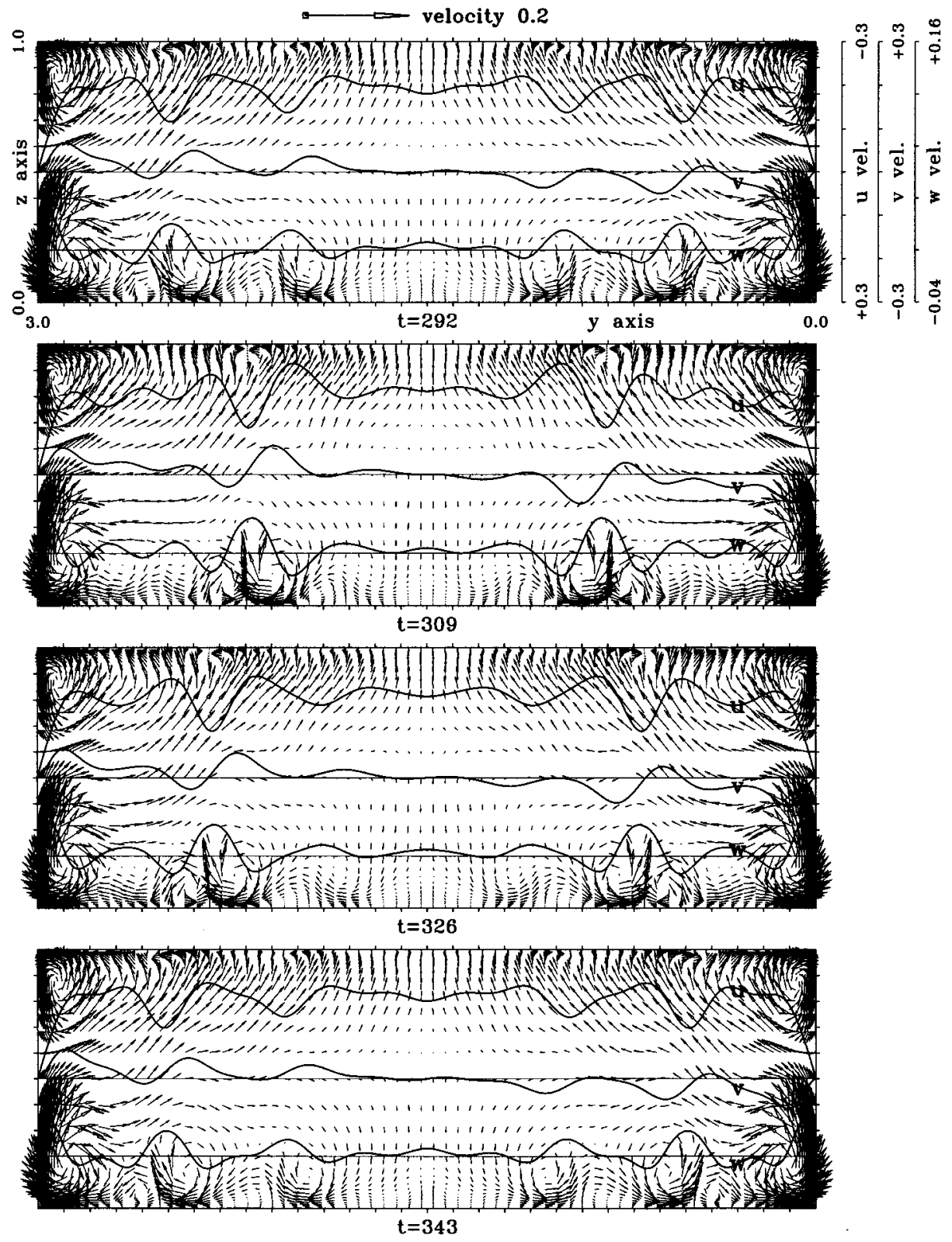

(a) the 1 st mode with periodicity 50

Figure 18. Illustration of time-varying TGL vortices at $x=0.525$ plane for $R e=1300$ : (a) first mode, with periodicity 50; (b) second mode, with periodicity 72 . The full lines denote the velocity components $u, v$ and $w$, computed at $z=0.04$, against $0 \leq y \leq 3$ 

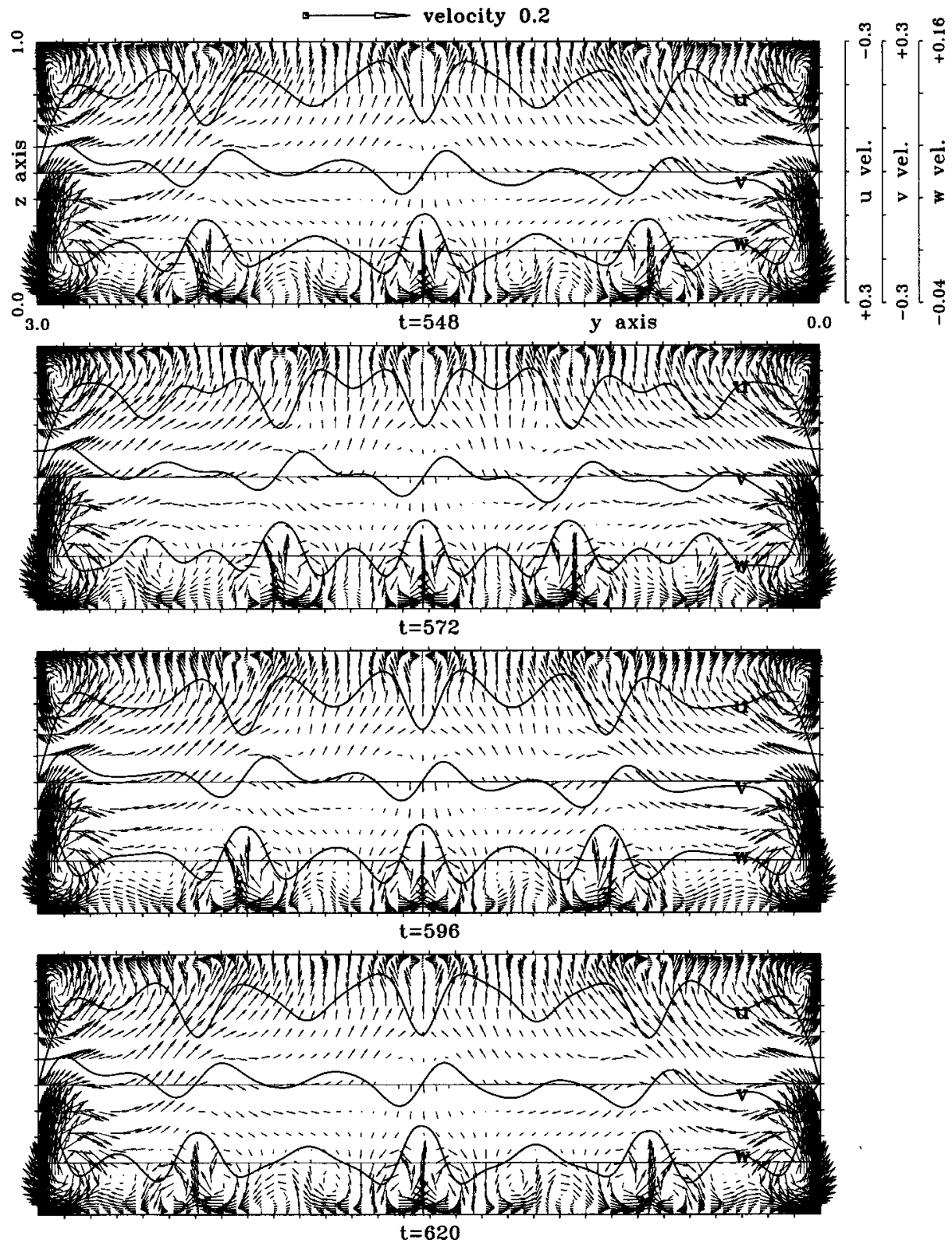

(b) the 2nd mode with periodicity 72

Figure 18 (Continued)

$(0.525,1.5,0.04)$ and plot them against time in Figure 19 , from which we realize that there exist two fundamental modes of fluctuations. In the beginning the period of fluctuation is 50 (Figure 18(a)), which is similar to that found in the case of $R e=1250$, and this is followed by a transitional period. At times beyond 550 the periodicity takes another value, namely 72 (Figure 18(b)), which is the same as that for $R e=1500$ [27]. 


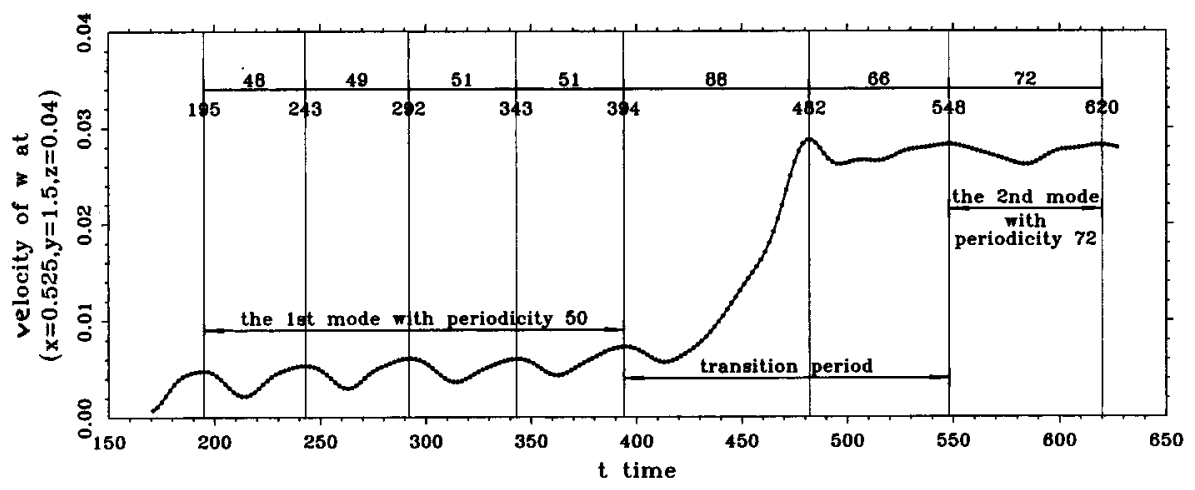

Figure 19. Time history of $w$-velocity $(150 \leq t \leq 650)$ at reference point $(0.525,1.5,0.04)$ for $R e=1300$

\section{CONCLUDING REMARKS}

In this paper we have applied a QUICK-type advection scheme and a finite volume method to explore in depth the change in the flow physics with the Reynolds number in a lid-driven rectangular cavity. The formulation uses primitive variables on the staggered grid. To accurately simulate the inherent physics, it is best to carry out a laminar flow analysis in flow domains covered with rectangular and non-uniform grids. The algebraic equations are thus exempt from the necessity of dealing with metric tensors and turbulence modelling. The predicted physics can be less contaminated by numerical errors, and solutions can be acquired that can well mimic the realistic flow physics. Based on the Reynolds numbers investigated and the prediction solutions obtained, some important findings are summarized in the following paragraph.

Inside the lid-driven cavity the corner eddies adjacent to the bottom plane become well established at $R e=50$. The corner eddies near the lid plane are hardly visible until the Reynolds number reaches 100. It is at this Reynolds number that the tube-type zero spanwise velocity forms. For Reynolds numbers larger than 300 the surface separating the primary core and the DSE/USE starts to detach from the upstream side-wall. In the course of flow evolution the fluid flow can reach its own steady state in cases where the Reynolds number takes on values which are smaller than 1000. Beyond this critical Reynolds number the flow field continues to be destabilized by the increasing alignment between the contour line of $v=0$ and the streamline. This destabilizing mechanism leads to a wavy surface of the spanwise velocity contour $v=0$ on the upstream side. As long as the Reynolds number is less than 1200, the sizes of both the USE and DSE remain steady. At $R e=1250$ the size of the USE starts to show a wavy phenomenon. Upon increasing the Reynolds number to 1300, the TGL vortices burst.

\section{ACKNOWLEDGEMENT}

This work was supported by the National Science Council of Taiwan under Grant No. NSC86-2611-E-002-021.

\section{REFERENCES}

1. M. Deville, T.-H. Lê and Y. Morchoisne (eds), Numerical Simulation of 3-D Incompressible Unsteady Viscous Laminar Flows, NNFM Vol. 36, 1992. 
2. O.R. Burggraf, 'Analytical and numerical studies of the structure of steady separated flows', J. Fluid. Mech., 24, $113-115$ (1966).

3. J.R. Koseff and R.L. Street, 'Visualization studies of a shear driven three-dimensional recirculating flow', in Three Dimensional Turbulent Shear Driven Flow, ASME, New York, 1982, pp. 23-31.

4. J.R. Koseff, R.L. Street, P.M. Gresho, C.D. Upson, J.A.C. Humphrey and W.M. To, 'A three-dimensional lid-driven cavity flow: experiment and simulation', Proc. 3rd Int. Conf. On Numerical Methods in Laminar and Turbulent Flow, Seattle, WA, August 1983, pp. 564-581.

5. J.R. Koseff and R.L. Street, 'Visualization studies of a shear driven three-dimensional recirculating flow', ASME J. Fluids Engng., 106, 21-29 (1984).

6. J.R. Koseff and R.L. Street, 'On end wall effects in a lid-driven cavity flow', ASME J. Fluids Engng., 106, 385-389 (1984).

7. J.R. Koseff and R.L. Street, 'The lid-driven cavity flow: a synthesis of qualitative and quantitative observations', ASME J. Fluids Engng., 106, 390-398 (1984).

8. C.J. Freitas, R.L. Street, A.N. Findikakis and J.R. Koseff, 'Numerical simulation of three dimensional flow in a cavity', Int. j. numer. meth. fluids, 5, 561-575 (1985).

9. C.J. Freitas and R.L. Street, 'Non-linear transport phenomena in a complex recirculating flow: a numerical investigation', Int. j. numer. meth. fluids, 8, 769-802 (1988).

10. A.K. Prasad and J.R. Koseff, 'Reynolds number and end-wall effects on a lid-driven cavity flow', Phys. Fluids A, 1, 208-218 (1989).

11. C.Y. Perng and R.L. Street, 'Three-dimensional unsteady flow simulations: alternative strategies for a volume averaged calculation', Int. j. numer. meth. fluids, 9, 341-362 (1989).

12. Y. Zang, R.L. Street and J.R. Koseff, 'A dynamic mixed subgrid-scale model and its application to turbulent recirculating flows', Phys. Fluids A, 5, 3186-3196 (1993).

13. C.K. Aidun, N.G. Triantafillopoulos and J.D. Benson, 'Global stability of a lid-driven cavity with throughflow: flow visualization studies', Phys. Fluids A, 3, 2081-2091 (1991).

14. O.A. Ladyzhenskaya, Mathematical Problems in the Dynamics of a Viscous Incompressible Flow, Gordon and Breach, New York, 1963.

15. S.V. Patankar, Numerical Heat Transfer and Fluid Flow, McGraw-Hill, New York, 1980.

16. S. Abdallah, 'Numerical solution for the incompressible Navier-Stokes equations in primitive variables using a non-staggered grid, II', J. Comput. Phys., 70, 193-202 (1987).

17. B.P. Leonard, 'A stable and accurate convective modeling procedure based on quadratic upstream interpolation', Comput. Meth. Appl. Mech. Engng., 19, 59-98 (1979).

18. C.R. Ethier and D.A. Steinman 'Exact fully 3D Navier-Stokes solutions for benchmarking', Int. j. numer. meth. fluids, 19, 369-375 (1994).

19. H.C. Ku, R.S. Hirsh and T.D. Taylor, 'A pseudospectral method for solution of the three-dimensional incompressible Navier-Stokes equations', J. Comput. Phys., 70, 439-462 (1987).

20. Y. Kato, H. Kawai and T. Tanshashi, 'Numerical flow analysis in a cubic cavity by the GSMAC finite element method', JSME Int. J. Ser. II, 33, 649-658 (1990).

21. A.B. Cortes and J.D. Miller, 'Numerical experiments with the lid-driven cavity flow problem', Comput. Fluids, 23, 1005-1027 (1994).

22. V. Babu and S.A. Korpela, 'Numerical solutions of the incompressible, three dimensional Navier-Stokes equations', Comput. Fluids, 23, 675-691 (1994).

23. M. Arnal, O. Lauer, Z. Lilek and M. Peric, 'Prediction of three-dimensional lid-driven cavity flow', in Reference 1 , pp. $13-24$

24. A. Kost, N.K. Mitra, M. Fiebig and R.U. Bochum, 'Numerical simulation of three-dimensional unsteady flow in a cavity', in Reference 1, pp. 79-90.

25. J.D. Bozeman, 'Numerical study of viscous flow in a cavity', J. Comput. Phys., 12, 348-363 (1973).

26. U. Ghia, K.N. Ghia and C.T. Shin, 'High-Re solutions for incompressible flow using the Navier-Stokes equations and a multigrid method', J. Comput. Phys., 48, 387-411 (1982).

27. T.P. Chiang, R.R. Hwang and W.H. Sheu, 'Finite volume analysis of spiral motion in a rectangular lid-driven cavity', Int. j. numer. meth. fluids, 23, 325-346 (1996). 